\title{
Sukzession und Tradition in
}

\section{Antike und Urchristentum}

\author{
E Bammel \\ University of Cambridge
}

\begin{abstract}
Succession und tradition in Antiquity and Early

Christianity

This article focusses on the concepts which today are known as 'succession' and 'apostolic tradition'. It investigates the meaning and reference of these concepts within the context of textual evidences from Antiquity and Early Christianity. From this historical perpective, the article aims at arguing that both concepts should primarily be understood within a judicial and political realm and not merely as theological ideas.
\end{abstract}

Wer die Peterskirche in Rom betritt, dessen Auge wird von dem Fries in der Apsis in den Bann gezogen, der Inschrift, die auf Griechisch und Lateinisch verkündet: Du bist Petrus....Der Gedanke wird ihn durchzucken, was mit dem Wort hier gemeint ist - an diesem Orte in goldenen Lettern eingegraben muss es sich auf die Cathedra der Weltkirche, deren Verbringung nach Rom und die Nachfolge Petri, des Stuhlinhabers in den römischen Bischöfen beziehen - und welches der ursprüngliche Sinn war.

1. Die uralte Streitfrage über den Nachfolger beginnt mit einem winzigen Belegstück. Matthäus 16:18f spricht in der Fassung, in dẹr die Stelle vorliegt, von etwas, das Simon Jona gegeben wird, und zwar als Antwort auf dessen Proklamation: Du bist der Christus: Derjenige, dem der Vater im Himmel eine Offenbarung zuteil werden liess, ist wert, seinerseits etwas zu erhalten und mitzuteilen, und zwar die 
Schlüssel des Himmelreichs. Wie die Offenbarung etwas ganz persönlich Gegebenes ist, so sind die Schlüssel etwas, das einem bestimmten Menschen übermacht worden ist. Die consecutio geht von Matthäus 16:17 unmittelbar hinüber zu Matthäus 16:19. Matthäus 16:18, in dem die Worte: Tu es Petrus stehen, fehlt im Text Tatians ${ }^{1}$ und ist, wie der Vers steht, das Ergebnis einer Erweiterung, die in Schüben erfolgt ist. Es handelt sich um:

* die Verleihung eines neues Namens, wie dies für die Jüngerschaft Jesu und schon des Täufers nicht untypisch war ${ }^{2}$ - er wird Simon bei anderer Gelegenheit als Beiname gegeben worden sein

- eine Aussage über die Kirche oder Gemeinde, etwas, das sich mit Matthäus 16: 18 a verbinden liess ${ }^{3}$ und

- die Grundbefindlichkeit der neuen Organisation (Matt 16:18c)

Die Verse sprechen auch in der jetzigen Form nicht von Nachfolge. Im Gegenteil: Der Bezug auf die Pforten der Hölle, die sich öffnen und das Erdreich überfluten werden, ${ }^{4}$ etwas, das der Verfasser als nahe bevorstehend angenommen haben muss, schliessen diese Möglichkeit aus.

Um diese Verse mit einem Nachfolger beziehungsweise der Nachfolge zu verbinden, bedurfte es einer völligen Neuinterpretation der Schlüsselbegriffe $\delta \dot{\omega} \sigma \omega$,

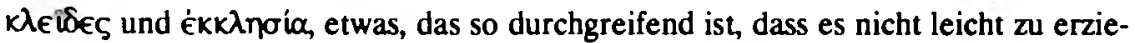
len war und nur in einem Bereich und unter Umständen mit Erfolg durchgeführt werden konnte, wo die Assoziationen, die jetzt fest mit den Versen verbunden sind, bereits aus anderen Gründen vorgebildet waren und für eine neue Anwendung bereitstanden.

In der Tat, die Stelle wird in den ersten Generationen christlicher Geschichte kaum je erwähnt. ${ }^{5}$ Roms Ansprüche wurden, sobald sie sich einstellten, ohne Bezugnahme auf diese Verse aufgestellt.

2. Versucht man weiterzukommen, so legt sich die Annahme des Einflusses priesterlicher Tradition aus dem Alten Testament nahe. Katholische Forscher waren gerne bereit, das miteinzubeziehen. Als entscheidend sahen das priesterliche Erbe A Ehrhardt ${ }^{6}$ und vor allem E Stauffer ${ }^{7}$ an.

Die Priester waren ein Stamm im israelitischen Verband, dessen Angehörige in Standmannschaften im Wechsel am Altar dienten. Das hochpriesterliche Amt wurde bis an den Anfang der herodianischen Zeit im Wege des Erbgangs weitergegeben, während es von da ab als 'Jahramt' zwischen den Gliedern von drei oder vier Geschlechtern hin - und herging. Deren Ansprüche wurden so mit einander zum Ausgleich gebracht.8 
In der Tat, so manche Einzelnachricht in frühchristlichen Quellen weist in diese Richtung. Von Johannes heisst es, dass er das Petalon, das hochpriesterliche Stirnband getragen habe. ${ }^{9}$ Über Jakobus berichtet man, dass er das weisse Gewand des Leviten angelegt habe und es ihm als einzigen gestattet gewesen sei, das Heiligtum zu betreten. ${ }^{10}$ Die Leitung der Kirche in Jerusalem war ein Amt, das in der Familie Jesu weitergegeben wurde, solange Mitglieder derselben verfügbar waren. ${ }^{11}$ Die Ansicht, dass das Papsttum in Rom wenig anderes ist als eine Übertragung dessen, was zuvor in Jerusalem in Geltung gewesen war, legt sich nahe. Ist doch die Thiara des Papstes der Krone, die der Hochpriester trug, nachgebildet. Freilich, es stellt sich die Frage, ob eine direkte Übertragung nachweisbar ist, eine Verbindung etwa der Art, wie sie entstand, als die platonische Philosophenschule von Athen nach Seleukia auswanderte. Die soeben herangezogenen Belege - es sind solche, die nicht aus Rom herrühren - weisen nicht über das dritte Jahrhundert zurück, während die älteren Quellen die Frage nicht berühren oder von priesterlichen Vorstellungen in einer allegorischen Weise Gebrauch machen. So scheint es doch, dass die priesterlichen Züge nicht viel mehr sind als Farbtupfer auf ganz anders gearteten

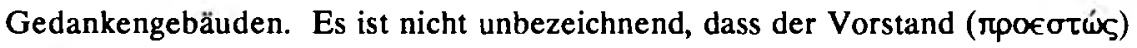
beim Abendmahl, wie Justin es beschreibt, kein Priester war. ${ }^{12}$ Quellen sind oft zufällig und geben dort, wo sie vorliegen, nicht immer die Wirklichkeit zureichend wieder. So mag es sein, dass priesterliche Traditionen eine stärkere Wirkung auf die römische Kirche ausgeübt haben, als die Quellen zum Ausdruck bringen. Doch werden sie kaum von entscheidender Bedeutung gewesen sein.

Auf der anderen Seite war ein Seitenzweig priesterlicher Tradition sicherlich für die Kirche Roms von Bedeutung. Es ist die Wirklichkeit und der geistige Anspruch des heidnischen Pontifex Maximus, der in derselben Stadt seines Amtes waltete. Wie sehr das der Fall war, erhellt aus einer Bemerkung Tertullians, der Kallist mit Hohn übergiesst, weil er ein überhebliches Edikt ganz in der Weise des Pontifex Maximus verlautbart habe. ${ }^{13}$

3. Eine weitere Möglichkeit der Erklärung stellt sich, wenn man die griechischen und jüdischen Schulen in Betracht zieht. Sind sie doch ihrer Natur nach Bildungen, die dazu da sind, einem kommenden Geschlecht zu Diensten zu sein und bald dazu neigen, sich zu Einrichtungen mit dem Anspruch auf Dauer zu entwickeln. Da eine Abhängigkeit Roms von den jüdischen Schulen ${ }^{14}$ kaum wahrscheinlich ist, genügt es, sich mit den griechischen zu befassen. Reiche Belege gibt es für die philosophischen Schulen - vier an der Zahl - in Athen. ${ }^{15}$ Wie wurde der Übergang von einer Generation zur nächsten bewcrkstelligt? In aller Regel behält das Haupt einer 
Schule seine Stellung auf Lebenszeit. Dass Chrysipp sich erkühnte (غ̇áppnoєv), zu Lebzeiten seines Lehrers Kleanthes eine eigene Schule zu bilden, war durchaus ungewöhnlich: Er musste im Freien beginnen. ${ }^{16}$ Diogenes Laertius vermeldet denn auch, dass Lakydes, das vierte Schulhaupt ( $\sigma \times 0 \lambda a ́ p \times 0$ ) nach Plato, seit unvordenklichen Zeiten ( $\alpha \pi \alpha$ lwwos) der einzige war, der seinen Nachfolger zu Lebzeiten einsetzte und damit sein Amt aufgab - tatsächlich ernannte er sogar zwei Nachfolger. ${ }^{17}$ Lykon übergab sein Schule in seinem Testament namentlich genannten Gefährten ( $\phi \dot{\lambda}(\mathrm{\imath})$ und überliess es diesen, ein Schulhaupt zu bestimmen. ${ }^{18}$ In späteren Zeiten haben der römische Kaiser und die Stadt Athen einen gewissen Einfluss auf die Schulen genommen, die bis dahin private Einrichtungen gewesen waren. Sie taten es, indem sie Jahr für Jahr Geschenke gaben oder, indem sie besondere Lehrstühle errichteten. Marcus Aurelius begründete zwei Lehrkanzeln, eine davon für Rhetorik - der Inhaber wurde von ihm selbst bezeichnet -, ein zweite für Philosophie, deren Inhaber von den 'besten, tüchtigsten und weisesten in der Stadt' ausgewählt werden sollte - wer tatsächlich das Wahlrecht hatte, ist eine offene Frage. ${ }^{19}$ Diels $^{20}$ und Mommsen 21 befassten sich mit dem Bruchstück einer Inschrift aus Athen, die einen Teil eines Schreibens der Plotina Augusta an Hadrian aus dem Jahre 121 nach Christus enthält und das von einem Lehrstuhl handelt, bei dessen Vergabe der Kaiser ein Wort mitzureden hatte (welches genau die ihm zustehende Gerechtsame war, ist nicht deutlich). In diesem Falle wurde die Wahl selber von den Studenten vorgenommen, die sogar das Recht hatten, einen Professor abzusetzen, während der Lehrstuhlinhaber - hier zeigt sich die Hand des Kaisers - ein römischer Bürger zu

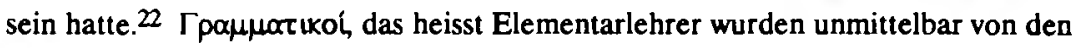
städtischen Behörden bestallt. Ein nach ordentlichen Regeln sich vollziehendes Verfahren war hinsichtlich der Schule unabdingbar, da Eigentum von erheblichem

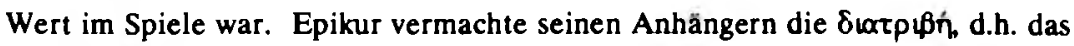
Schulgebäude in seinem Garten. ${ }^{23}$ Viele andere Geschenke folgten; bekannt sind die grosszügigen Gaben, mit denen Attalos die Akademie versah. Das Verfahren, nach dem ein Wechsel vollzogen wurde, war vorher festgelegt, im Einzelnen aber war es verschieden von Schule zu Schule und von Lehrstuhl zu Lehrstuhl.

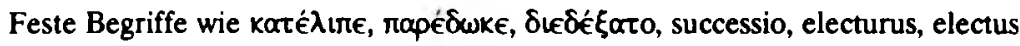
und selbst ordinatio finden in diesem Zusammenhang Verwendung. Dieselben Worte sind aus frühchristlichen Texten bekannt. Aber die Bedeutung ist nicht in jedem Fall dieselbe. Ordinatio etwa bezeichnet die Regel der Nachfolge und nicht den neuen Stand eines Einzelnen.

Wie auch immer die Nachfolgeregelung gewesen ist, sie scheint volle und durchgängige Beachtung gefunden zu haben. Indessen ist ein Fall bekannt, wo der Ausgang anders war: Der Nachfolger, den Proclus bestimmt hatte, wurde von seinen 
Schülern nicht hingenommen. Sie wählten statt dessen Isidorus und hatten mit dieser Handlung Erfolg. 24

Alowoxń war ein terminus technicus für eine bestimmte Schule. Erst in nachsinnender Betrachtung gestaltete sich daraus eine Verknüpfung der Einzelnachrichten und ein Stammbaum der Nachfolgeschaft für die verschiedenen Schulen. Sotion von Alexandrien, der zu Beginn des zweiten Jahrhunderts vor Christus wirkte, war der erste, der solche Ketten von Nachfolgeschaften oder Listen von Sukzessionen vorgeführt hat. 25 Es war jedoch nicht seine Absicht, die Glieder einer bestimmten Kette namhaft zu machen. Vielmehr ging es ihm darum, soweit wie möglich Ordnung in das riesige und schon zerfliessende Material von Worten und Szenen, die diesem oder jenem zugeschrieben wurden, zu bringen. $\mathrm{Er}$ ist, man ist versucht zu sagen, der Erfinder der übergreifenden Traditionsketten. Wahrscheinlich war er nicht unbeeinflusst von dem, was gleichzeitig in der politischen Welt vorging, der Errichtung von Staaten, die sich selbst in einer $\delta$ oofoxí stehend wussten. Die Traditionsketten wurden nach rückwärts verlängert, durch die Entdeckung der altpythagoräischen Schule durch die Alexandriner. ${ }^{26}$ Es ist bezeichnend für die so hergestellten Traditionen, dass sie von einer Wesensverwandtschaft auf eine persönliche Verbindung, eben auf eine $\delta$ coóx

Den Listen eignet kein spezifischer, insbesondere kein rechtlicher Wert. Für den Historiker ist ihre Bedeutung gleichermassen gering, wie dies schon Friedrich Nietzsche festgestellt ${ }^{27}$ und W. von Kienle durch neue Beweismittel ergänzt hat. ${ }^{28}$ Man mag sie mit den Listen der Irrlehrer bei den Kirchenvätern vergleichen. Sie haben teilweise demselben Zweck gedient, nämlich einen Hilfbeweis für die Gegenwart zu führen. Wie die Alexandriner mit ihren Listen nachweisen, dass ihre Philosophie älter sei als die der Barbaren ${ }^{29}$, so erheben die Väter aus den Ketzertabellen, dass deren Irrlehren ihren Ursprung in der heidnischen Philosophie und nicht im Christentum haben. ${ }^{30}$ Diogenes Laertius will darüber hinaus das unwiderrufliche Ende der Tradition aufzeigen. Der Schatz ist verloren und die Versuche auf Seiten der Römer, ihn wiederzugewinnen, werden von keinem Erfolg gekrönt sein: Sind sie doch selbst nicht $\delta$ rádoxol sondern - kann man Abwertenderes sagen? blosse ériyovo ${ }^{31}$.

Was ergibt sich daraus? Der Begriff $\delta$ woooxór der von Aristoteles, wenngleich in einer weiteren Bedeutung, ${ }^{32}$ zum ersten Mal gebraucht worden war, wurde in der hellenistischen Zeit ein beliebter Ausdruck, der verwendet wurde, um zusammenzubringen, was in den Quellen getrennt von einander aufzufinden war. Das geschah dann freilich in verschiedener Weise und konnte von einer neuen Bewegung nicht ohne weiteres als Muster in Verwendung genommen werden. 
Sukzession hat es auch - ob nach dem Vorbild der Philosophenschulen oder davon unabhängig, sei dahingestellt - in der Welt der Juristen gegeben. Pomponius (2. Hälfte des 2. Jhdts n. Chr.) macht Angaben (Dig. 1,2,2, 47-53) über die beiden römischen Rechtsschulen (sectae), die jedenfalls bis in die tiberianische Zeit ${ }^{33}$ zurückreichen, aber schon in der Mitte des zweiten Jahrhunderts aufgehört haben oder zur Bedeutungslosigkeit herabgesunken sind, 34 bezeichnet den Gründer als auctor, zeigt den Wechsel von einem Schulhaupt zum anderen durchweg durch successit an und erwähnt, dass der Nachfolger ein solcher war, der zuvor audivit, um dann in die Lage zu kommen, selbst zu respondere. Man hat darin den Hinweis auf eine genossenschaftliche Form gesehen und die Vornahme des Wechsels durch Wahl angenommen. 35

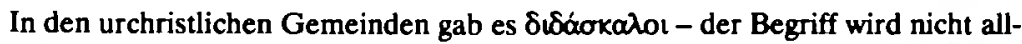
zu oft verwendet. Ganz überwiegend werden sie neben anderen Amtsträgern erwähnt. Der älteste Beleg, die Quelle, die von der Ausbreitung des Evangeliums

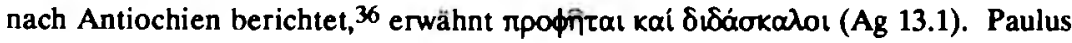

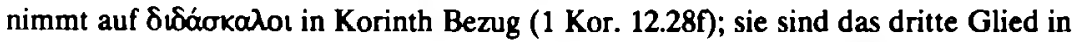
einer Liste, die mit dem herausragenden Amt des Apostels einsetzt. Anderswo wird der Begriff zusammen mit roujéveৎ verwendet (Eph. 4.11). Die Tätigkeit des Lehrers wird als die Darreichung von Milch beschrieben, als etwas, das durch anderes ergänzt werden muss (Hebr. 5.12ff).

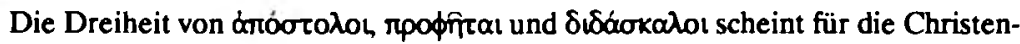
heit von entscheidender Bedeutung gewesen zu sein. Weil es eine Trias war, musste es schwer, wenn nicht unmöglich sein, zu einer Nachfolgeordnung zu kommen. Das spricht gegen die Ansicht, dass die Analogie der Schule und ihrer Soofoxal einen durchgreifenden Einfluss auf das Christentum ausgeübt hat. Harnacks abwăgendes Urteil hat seine Gültigkeit nicht verloren: Der Vergleich mit den philosophischen Schulen sei fast ganz aus apologetischen Gründen vorgenommen worden, anders dagegen möge es sich bei einzelnen christlichen Lehrern verhalten haben; aber diese standen gewissermassen neben der griechischen Christenheit'. ${ }^{37}$

Die Überlieferung, dass der Herrenbruder Jakobus einen $\theta$ póvos, das heisst einen Lehrstuhl hatte - zur Zeit des Julius Africanus war sie bekannt ${ }^{38}$ - ist eher eine romantische Verklărung als eine glaubwürdige Nachricht über das, was tatsächlich im ersten Jahrhundert in Geltung war.

4a. Die Ausführung des Kults in geregelter Ordnung ist einer der Züge, der sich dem Beobachter der Religionen der Alten einprägt. Dazu gehört die Abfolge von einem Geschlecht zum anderen. Oft werden die Priestertümer vererbt, aber auch 
da, wo eine andere Ordnung in Geltung steht, gilt sie als unverbrüchlich, ja heilig. Wie aber verhielt es sich mit Religionen, die nicht zum alten Bestand eines Landes gehörten, sondern von aussen hereinkamen? Wusste man um deren Stetigkeit? War da nicht Gefahr, dass in ihnen Unordnung überhand nahm, sich ein Usurpator aufwarf oder gar etwas Neues, sogar dieser Religion Fremdes aufs Tapet gebracht wurde?

In Aegypten, wo man die Dinge mit bürokratischer Gründlichkeit regelte und wo es, wie W. Otto sich ausdrückte, schon eine Staatskirche gegeben hat, ${ }^{39}$ ist man auf dieses Problem gestossen und ihm zuleibe gegangen. 1916 veröffentlichte W. Schubart einen - später nach ihm benannten - Berliner Papyrus aus dem Anfang des zweiten Jahrhunderts vor Christus, ${ }^{40}$ der sich mit dieser Frage beschäftigt. Danach wurde es dem neu ins Amt tretenden Priester der unter den Ptolemäern aufgeblühten Dionysusreligion zur Pflicht gemacht, innerhalb einer kurz bemessenen Zeitspanne nach Alexandrien zu fahren und dort den Nachweis darüber zu erbringen, von wem er das Priestertum übertragen erhielt und von wem dieser sein Vorgănger das Priestertum erworben hatte. Eine Sukzessionsliste wurde verlangt, ohne $Z$ weifel in Alexandrien aufgehoben und mit dem dort bereits vorhandenen

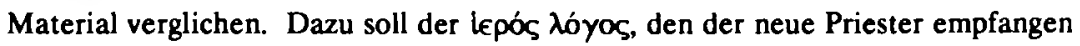
hat, versiegelt eingegeben und mit seinem Namen bezeichnet werden. ${ }^{41}$

Die Folge vom Vater auf den Sohn, die gerade bei den ägyptischen Priestertümern die Regel war, ${ }^{42}$ wird nicht gefordert, wohl aber eine geregelte Abfolge, wie immer sie sich im Einzelnen gestaltet haben mag.43 Da sie sich nicht in der natürlichen Weise der Geschlechterfolge vollzog, war es notwendig, Namen zu nennen, um der tatsächlich erfolgten Übertragung ganz sicher zu sein. Auch musste es möglich sein, im Falle des Falles den Inhalt der Lehre zu erfahren, um Betrügereien aufzudecken. Philopator IV, aus dessen Zeit das Edikt stammt, war selbst ein grosser Verehrer des Dionysus.44 Dennoch gab er seıner Religion nicht alle und jede Freiheit, sondern sah sich genötigt, nach Grundsätzen zu verfahren, die in seinem Königreich üblich waren und diese auf die neue Lage umzuschreiben. Mag das Misstrauen der Amtsträger der einheimischen Religion gegenüber der geheimnisumwitterten Welt des Dionysuskults eine Rolle gespielt haben, in der Hauptsache handelt es sich um die Anwendung des alten Prinzips auf ein neues Phänomen.

Wie sich die Sache von seiten der Anhänger her darstellte, erhellt aus einer Inschrift aus Delos, die sogar etwas älter als der eben besprochene Papyrus ist. ${ }^{45}$ Daraus ergibt sich: Der Serapionkult hatte auf der stark unter ägyptischem Einfluss stehenden Insel Eingang gefunden. Der Dienst wurde dargebracht, aber es war den Anhängern mindestens zwei Generationen hindurch nicht möglich gewesen, eine Kultstätte, ein Serapion zu errichten. Schliesslich gelang es. Da wurde alles durch 
eine Klage von Gegnern vor Gericht wieder infrage gestellt. Aber 'der Gott siegte', wie er es seinem Priester im Traum vorausgesagt hatte. Diesem Sieg zu Ehren wurde die Inschrift aufgestellt. Sie zeigt deutlich die Animosität gegen nichteinheimische Kulte, wie sie sogar an diesem Umschlagplatz des Handels bestand. Aber sie zeigt noch mehr: der Priester, der die Inschrift setzte, weist ausdrücklich darauf hin, dass er Nachfolger eines anderen und dieser wieder eines anderen, der aus Ägypten auf die Insel kam, ist. Die Angabe und ihre Hervorhebung auf einer Inschrift zeigen, dass es den Anhängern des Kults selbst darum zu tun war, eine Sukzessionsliste vorzulegen, Bestăndigkeit aufzuweisen und damit den Anschein der Zuverlässigkeit zu geben - vielleicht war die Formulierung zuerst für die Gerichtssitzung geprägt worden, in der es um das Recht der Gemeinschaft gegangen war. In diesem Falle sind die drei namentlich genannten Priester Grossvater, Vater und Sohn, d.h. die Praxis der ägyptischen Religion war von der Serapionsgemeinde, in der sie an sich nicht in Übung stand, übernommen worden, um Eingang und Ansehen zu erlangen. In der Ferne, wo Misstrauen bestand, war es noch mehr als anderswo notwendig, die Kontinuität in den Vordergrund zu rücken.

Wie man sich die Handlungsweise des ägyptischen Hofs vorstellte, zeigt die von Josephus überlieferte Geschichte über den vor dem Konig ausgetragenen Streit zwischen den in Alexandrien lebenden Juden und Samaritanern über den Vorzug der Tempel, denen sie verpflichtet waren. Die Juden weisen für Jerusalem auf die

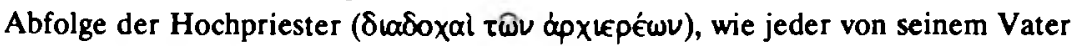
die Würde erhalten und den Vorstand des Tempels gebildet habe (Ant. XIII,78), hin und vermögen damit, den den Wettstreit entscheidenden Eindruck zu machen.

4b. Gilt das, was sich im griechischen Osten als notwendig herausstellte, auch für den Westen? Bezeichnend für die Einstellung Roms ist die Behandlung des Kults der Bacchanalien. Zu Beginn des zweiten Jahrhunderts vor Christus eingefuhrt durch einen Griechen von geringer Herkunft ${ }^{46}$ - nicht einmal sein Name wird in den Quellen genannt - haben die Bacchanalien zu Ausschweifungen in Rom geführt, die die entschlossene Unterdrückung durch den Senat notwendig machten. Eine Ausnahme wird für Italien ausserhalb Roms ${ }^{47}$ nur insoweit gemacht, als eine geweihte vetusta ara aut signum vorhanden war. Was gegen den Kult sprach, war also die Unbekanntheit desselben am Ort wie die Person, die ihn verbreitete.

Die Unterdrückung war ein beispielgebendes Ereignis - darum der überaus ausführliche, aus zwei an sich von einander unabhängigen Teilen zusammengesetzte Bericht bei Livius. ${ }^{48}$ Die eingeschränkte Erlaubnis ist es ebenso. Die Massnahme blieb kein für sich stehendes Ereignis, sondern wurde als Muster für die Hand- 
habung ähnlich gelagerter Fälle verwendet. Die Kontrolle wurde sogar verstärkt. Cäsar verbot alle collegia praeter antiquitus constituta. ${ }^{49}$ Augustus schritt gegen sie alle praeter antiqua et legitima ein. ${ }^{50}$ Die Sprache des Senatsbeschlusses gegen die Bacchanalien ist unverkennbar. Die Spaltungen, die für ein collegium novum, seine Entstehung ${ }^{51}$ und seine Fortexistenz, 52 bezeichnend sind, werden als Grund angegeben. Die wiederholte Einschärfung weist andererseits auf die Schwierigkeit der Durchsetzung hin.

Die Quellen berichten uns ihrer annalistischen Natur gemäss nur über das Endergebnis. Jedoch muss anderes vorausgegangen sein: Eine Untersuchung durch einen städtischen Amtsträger, vielleicht auch ein Beschluss des Senats. 53 Solche Verfahrensweise ist erst recht für die spätere Zeit anzunehmen, als Rom Hauptstadt eines Reichs geworden war und es galt, einerseits Rücksichten zu nehmen, andererseits aber den besonderen Charakter der urbs zu wahren. Aus dem Augenblick geborene und nicht in der üblichen Verfahrensweise verankerte Massnahmen des praefectus urbi sind, so wenig man sie ausschliessen kann, doch unwahrscheinlich.

Es ist denn auch nicht nur eine Massnahme ergriffen worden. Neben der Ausweisung hat es die Wegräumung der Kultmittel überhaupt oder doch von öffentlichen Orten gegeben. ${ }^{54}$ Das lässt auf eine nicht ganz summarische Behandlung, auf verschiedene Befunde, eben auf eine vorausgegangene Untersuchung schliessen. Gab es eine solche, so müssen sich gewisse Verfahrensweisen bei den Behörden, den Aedilen oder dem Fremdenprätor, je nachdem wie der Fall beschaffen war, herausgebildet, aber auch Verhaltensweisen bei den Betroffenen beziehungsweise deren Rechtsvertretern ergeben haben. Das gilt, auch wenn es, wie es im Wesen des Koerzitionsverfahrens lag, keine eigentlichen Strafprozesse gegeben hat. Es sind vielmehr Voruntersuchungen anzunehmen; vergleichbar dem, was als elogium zu Anfang des Strafprozesses zusammengefasst wurde. Andererseits gab die Ausnahme, die der Senat im Falle der Bacchanalien zugestand, ${ }^{55}$ eine Handhabe für die Klărung des Sachverhalts. Der Nachweis eines von altersher bestehenden Kults war durch Antrag beim praetor urbanus, der den Senat unterrichtet, zu erbringen. Das Verfahren ermöglichte dann auch eine Feststellung zur Person.

Die Kulte hatten nach den alle früheren Forschungen aufhebenden Ergebnissen von Ludwig Schnort von Carolsfeld ${ }^{56}$ und Gerda Krüger ${ }^{57}$ in der prinzipalen Zeit die Form von collegia, die die Rechtsfähigkeit besassen und darum eine andere Behandlung, als die Räuber sie erfuhren, 58 erhielten. Das gilt auch für die Christen. 59

Ein Kriterium wurde bei der Behandlung der Bacchanalien festgelegt: das Alter eines Kults - was dem nicht entsprach, war verdächtig und wurde gleich unterdrückt. Wie aber konnte man sicher sein, dass das, was sich als alt ausgab, es auch 
wirklich war? Ist denn nicht der Wechsel, das Einströmen neuer Elemente und ihre Amalgierung das für die Religionen der Randsiedler Typische? So wurde es die Pflicht der Behörden, darüber zu wachen, dass der Gesichtspunkt des Alters, der eine Duldung möglich machte, auch weiterhin gültig war. Andererseits aber sah der im Verdacht stehende Kult sich veranlasst, die Kontinuität unter Beweis zu stellen. Was konnte geschehen? Lehren waren nur, soweit sie das Sittliche betrafen, von Belang. Viel grösseren Erfolg musste es versprechen, wenn es gelang, das Alter des Kults und möglichst auch sein Vorhandensein in Italien von altersher aufzuweisen. Für den Kultdiener aber war es erforderlich, die eigene Person aus der Anonymität herauszuheben, sie als bekannt und eingefuihrt darzustellen - das geschah am besten durch den Hinweis auf einen Gewährsmann. Keiner war dazu besser geeignet als der Vorgănger, in dessen Fusstapfen man getreten war. Für die Erhebung von beide aber war die Durchsichtigkeit der Verhältnisse, das, was im Zusammenhang mit den Bacchanalien aperta religio genannt wird, ${ }^{60}$ von ausschlaggebender Bedeutung.

So waren die allgemeinen Verhältnisse in Rom, die Verfahrensweise der Behörden und die Gesichtspunkte bei der Untersuchung derart, dass sie von denjenigen im Osten nicht wesentlich abwichen. Darüber hinaus: es ist nicht unmöglich, dass die römische Verwaltung wie in anderen Bereichen so auch in diesem die ägyptische sich zum Muster nahm.

5. Wie steht es um die christliche Gemeinde in Rom? Gibt es einen Hinweis dafür, dass sie sich innerhalb eines solchen Bezugssytems lebend wusste, dass sie sich dementsprechend verhielt und der Aussenwelt gegenüber darstellte? Der erste Klemensbrief scheint dafür einen Beleg abzugeben.

Der Brief schliesst mit einem hymnischen Gebet für den Herrscher, der auf Erden als das erscheint, was Gott im Himmel ist, und der Aufforderung, ihm in nichts zu widerstehen. ${ }^{61}$ Zuvor war seiner überhaupt nicht gedacht worden. Im Brief selber war Gehorsam nur den Oberen der Gemeinde gegenüber eingeschăft worden - ihnen zu widerstehen hiesse, sich Gottes Willen zu widersetzen. 62 Könnte dies dafür sprechen, dass das Kapitel über den Herrscher, zumal es in der Synagoge vorgeprägtes Gut aufnimmt, 63 ein Wildwuchs ist ${ }^{64}$, so hat es doch einen Bezug zum Anfang des Sendschreibens, wo nämlich ein Thema abgehandelt wird und Worte Verwendung finden, die sowohl einen kirchlichen wie einen politischen Bezug

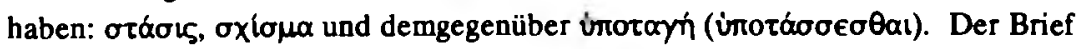
selbst führt dies auf breiter Front im Hinblick auf den Bestand und das Wohlergehen der christlichen Gemeinde aus. Zwiespalt innerhalb der Organisation aber hatte, wie aus den Parallelen erhellt, ein politisches Gesicht: er gefährdete die Dul- 
dung. Aus diesem Grunde wird die politische Loyalität der Christen so stark unterstrichen. Sie ist nur eine Anwendung des Gehorsamseifers, den schon die Väter (rartep€s) in ihrem ganzen Leben bezeigten.65 Nicht zufällig wird dieser Hinweis auf sie am Anfang des Gebets für den Herrscher wiederholt. Durchgehend ist die Gehorsamslinie in der Glaubensgeschichte. Im Vergleich zu ihr erscheinen die bedauerlichen Vorkommnisse in Korinth als klein. Kapitel 6.1,66 die einzige Stelle, wo zuvor auf Drangsal der Gemeinde beziehungsweise deren beider Gründer angespielt worden war, lässt durch die Wahl der für die Sinngebung richrungweisenden

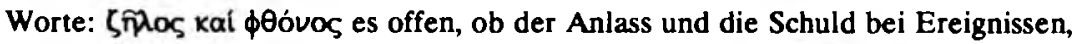
die sich aussen oder innen abgespielt haben, zu suchen ist. ${ }^{67}$ Der Verfasser ist also darum bemüht, die politische Macht nicht herauszufordern. So entsprechen Anfang und Ende einander: wie das Ende die Staatstreue der Christen in der bestimmtesten Weise herausstellt, so geht der Verfasser am Anfang und dann immer wieder mit starker Hand gegen die aufgetretenen Spaltungen vor; er tut es der möglichen politischen Folgen wegen. Man soll wissen, dass die Christen seit altersher willens und in der Lage sind, Ordnung zu schaffen. Es ist keine Frage: der Brief ist nicht ein rein innerkirchliches Dokument, nicht einfach ein Sendschreiben an eine andere Gemeinde oder unter diesem Titel ein Enzyklika, der es um die Ingeltungsetzung eines Anspruchs Roms geht, ${ }^{68}$ er ist vielmehr mit einem Seitenblick auf die 'Obrigkeit' verfasst. Insbesondere Anfang und Ende sind so in Worte gesetzt, dass sie vorgelesen und im Bedarfsfall gleichsam als ein Alibi den Behörden vorgewiesen werden konnten.

In diesem Zusammenhang ist auch zu sehen, was über die Verfassung der korinthischen Gemeinde gesagt wird. An sich ist die Gemeinde eine sich selbst verwaltende Einheit: Das $\pi \lambda \hat{\theta} \theta 0$ s entscheidet über die Streitfragen. 69 Aber es gibt ausserdem Ėifokorot kal $\delta$ tókovoh und zwar nicht erst seit kurzem sondern von altersher, wie durch ein aus Is. 60,7 zusammengebogenes Zitat bewiesen wird $(42,5)$. Ou katvāys ist der entscheidende terminus. Dem entspricht es, dass die gegenwärtige Ordnung als durch Nachfolge ( $\delta 1000 \times \gamma)$ erworben und in Übereinstimmung mit der Gemeinde hergestellt bezeichnet wird $(44,2) .70$

Die $\delta$ coofoxm ist das Element, das die Verbindung mit der Vergangenheit sicherstellt. Namen werden noch nicht genannt - die allgemeine Feststellung genügt eben. Im Bedarfsfall hätte man mehr sagen können.

Nachgeliefert werden Namen in der aus Irenäus von Euseb ${ }^{71}$ übernommenen Liste der römischen Bischöfe. Es sind zwölf Amtsträger, die, von den Gründern der Gemeinde abgehoben, aufgezahlt werden. Man hat die Liste bisher mangels einer Alternative als eine Einheit angesehen, ${ }^{2}$ obwohl doch Irenäus und ihm folgend Euseb schon einen Abstand zwischen den ersten, nämlich Linus, Anenklet und Cle- 
mens und den folgenden Namen deutlich markiert hatten, während eine Zahl nur

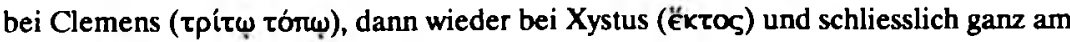

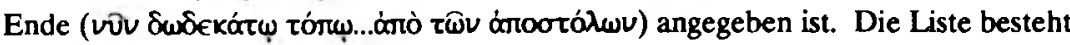
deutlich aus drei Teilen, einer bis auf Clemens reichenden und einer anderen mit Anicet abschliessenden Aufreihung, welcher letzteren die Bemerkung über den derzeitigen Bischof und dessen Vorgänger angehängt ist - die durchlaufende Numerierung ist das letzte, was hinzugekommen ist. Bei Irenâus gibt es nur die Hervorhebung des dritten, sechsten und zwölften Amtsträgers, während es sich bei Hippolyt ${ }^{73}$ um eine reine Personalliste handelt. Der Aufweis der Swowoxń konnte durch eine kurze Liste völlig ausreichend dargetan werden. Mit ihrer Hilfe konnte sichergestellt werden, dass ein bestehender Zustand nicht von ungefähr, sondern begründet war - deep rooted, wie der Engländer sagt. Die lange, bis an die Anfänge zurückreichende Iiste von zwőlf (!) Namen ist eine künstliche Konstruktion, die notwendig wurde, sobald mit dem Begriff $\delta$ la $\delta 0 x$ nicht eine rechtlich-politische Aussage gemacht, sondern eine theologische Absicht ganz bestimmter Art in Auge gefasst wurde. ${ }^{74}$ Die Listen bei Irenäus und Hippolyt lassen uns noch die Spuren der Anfänge 75 dieser Entwicklung erkennen.

Es waren drei Momente, die mehr oder weniger stark in den Vordergrund der Überlegungen der Behörden ${ }^{76}$ traten und dem zufolge die Selbstdarstellung neu auftretender Religionen bestimmten: das Alter $7 \boldsymbol{7}$ und die Bekanntheit des Kults, die Durchsichtigkeit des Dienstes und die Geordnetheit der Vorgänge innerhalb der Religionsgemeinschaft. Für das letztere war der regelgerechte Übergang von einem Gemeindevorstand zum anderen von ausschlaggebender Bedeutung.

Das christliche Schrifttum ${ }^{78}$ und die tatsächlichen Verhältnisse ${ }^{79}$ zeigen, dass man dieses Rahmens nicht uneingedenk war. So deutet das, was im Umfeld und in den Anfängen des Christentums sich als Sukzession darstellt, eher auf eine Bedeutung im rechtlich-politischen als im theologischen Bereich. Diese letztere ist erst hinzugekommen. 80

Man weiss wohl: alle Wege führen nach Rom, jeder für sich und ohne dass auf einen anderen ausgewichen werden müsste. Indessen: aller Rinnsale - ohne Ausnahme, die in den Tiber fliessen, bedurfte es, um seine Mühlen zu speisen und schliesslich das zu bewirken, was heute als die apostolische Tradition bekannt ist.

\section{FuBnoten}

1. A. Harnack, ZKG 4 (1880) S.471ff. - Zur Uneinheitlichkeit der Verse s. P. Hoffmann in J. Gnilka, Neues Testament und Kirche (= Festschr. R. Schnackenburg), Freiburg 1974, S.94ff. 
2. Mk 3.17; vgl. Lk 9.54. - Die Bezeichnung Jesu als đuuò̀s tỗ Oeỗ in John. 1.36 wird aus der Benennung als Lamm (im Unterschied zu den Donnersöhnen) durch den Täufer entwickelt worden sein.

3. P. Lampe. Das Spiel mit dem Petrusnamen NTSt 25(1978/9) S.227ff.

4. Das Bild ist hochapokalyptisch; s. E. Stauffer, Die Theologie des Neuen Testaments (Stuttgart 1941) S. 192.

5. W. Köhler, Omnis ecclesia Petri propinqua (Heidelberg 1938).

6. The Apostolic Succession (London 1953).

7. Zur Vor- und Frühgeschichte des Primatus Petri, ZKG 62 (143/4) S. $3 \mathrm{ff}$.

8. Verf, Judaica (Tübingen 1986) S.21ff. - Die Regelung ist mit den Verhältnissen in Rom zu vergleichen, wo fünf oder sechs gentes majores, von denen der princeps senatus genommen wurde, eine besondere Rolle spielten, wie Chr. Meier gezeigt hat (Gedenkschrift W. Kunkel, Frankfurt 1984, S. 192).

9. Euseb, hist. eccl. 5.24.3.

10. Euseb 2.23.6

11. Verf, Jesu Nachfolger (Heidelberg 1988) S.40f.

12. Justin, Apol. 67; vgl. Did.10.6

13. Audio etiam edictum esse propositum, et quidem peremptorium. Pontifex scilicet Maximus, quod est episcopus episcoporum, edicit: Ego etc. (De Pud.1).

14. Über sie s. die Tabellen in Strack-Billerbeck, Kommentar z. N.T. aus Talmud und Midrasch VI sowie W. Bacher, Tradition und Tradenten Leipzig 1914).

15. Grundlegend und bis zum heutigen Tage von niemand anderem auch nur entfernt erreicht ist K.G. Zumpts Darstellung 'Über den Bestand der philosophischen Schulen in Athen und die Succession der Scholarchen', Abhandlungen d. Kgl. Akademie d. Wissenschaften zu Berlin 1842 (Berlin 1844) S. 27ff; dort S. $116 \mathrm{ff}$ auch eine Tabelle über die Abfolge der Schulleiter.

16. Diog. VII.7 (185.179).

17. IV.8 (60).

18. 70. - Es liegt nahe, an Joh. 15.15 zu denken und in diesem und den folgenden Versen eine letztwillige Anordnung zu erblicken - Andererseits ernannte Straton den Lykon zum Nachfolger und forderte die anderen auf, damit übereinzustimmen.

19. Diels (s. nächste Anm.) spricht von 'Schulverhältnissen, die uns leider nur allzu unbekant sind'.

20. Archiv f. Geschichte d. Philosophie IV (1891) S. $486 \mathrm{ff}$.

21. Ges. Schriften III (Berlin 1907) S.50ff.

22. ...quae ad diadochos ordinationem pertinet... (Termini wie successor, diadochi, ad diadochen sectae Epicureae, diadoches begegnen in dem Brief). Es war die 
Absicht Plotinas, mit dem Schreiben an ihren Sohn eine Ausnahme für die Schule Epikurs zu erreichen; sie hatte damit Erfolg.

23. Dio. X 17. In diesem Fall war die Anordnung kompliziert: er gab sein Eigentum an zwei Personen mit der Auflage, dass sie den Peripatos dem Hermarch

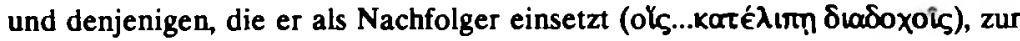

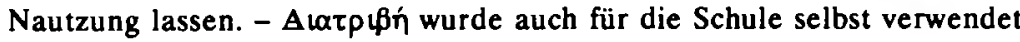
(Galen, De puls. diff. 2.4). - Die Schulen sind "mehr als Privatvereine" (Zumpt S.89).

24. Damascii Vitae Isidori Reliquiae ed. C. Zintzen (Hildesheim 1967) S. 292:

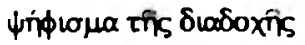

25. W. v. Kienle, Die Berichte über die Sukzessionen der Philosophen in der hellenistischen und spätantiken LIteratur (Diss. Berlin 1961) S.79ff. Sotion sind andere gefolgt; zu den Restnachrichten über sie s. Kienle S.92ff.

26. s.v.Kienle S. $58 \mathrm{ff} .98$.

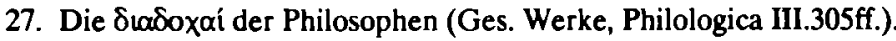

28. S.97ff.

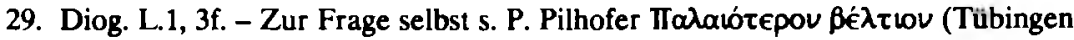
1991)

30. vgl. Ad. Hilgenfeld, Ketzergeschichte des Urchristenthums (Leipzig 1884).

31. Chrysipp war für ihn der letzte Philosoph, der diesen Namen verdiente.

32. v. Kienle 57.

33. W. Kunkel, Herkunft und soziale Funktion der römischen Juristen (Graz ${ }^{2}$ 1967) S.341.

34. Kunkel S. 342.

35. O. Lenel in: Enzyklopädie der Rechtswissenschaft I (München 1915) S. 362; R. Sohm, Institutionen (München 71923) S.97; J.K. Stirnimann, Die Praescriptio Tertullians im Lichte des römischen Rechts und der Theologie (Freiburg i. Ü. 1947) S. 164ff.; F. Wieacker, Recht und Gesellschaft in der Spätantike (Stuttgart 1964) S. 94ff.

36. vgl. Jeremias, ZNW 1938 S. $205 \mathrm{ff}$.

37. Mission und Ausbreitung ${ }^{4}$ (Leipzig 1924) S. 271.

38. Euseb., h.e. 7.19.

39. W. Otto, Priester und Tempel im hellenistischen Aegypten II, Leipzig 1908, S. 282 ff.

40. Amtliche Berichte aus den KgL Kunstsammlungen (= Bleiblatt zum Jahrbuch der Kgl Preuss. Kunstsammlungen) 38 (1916/17) Sp. 189 ff. 
41. Schubarts eigene Erklärung (Sp. 195), dass diese Anordnung in der Absicht erfolgt sei, um die Mysterienlehre in einer orthodoxen Form für das ganze Reich durchzusetzen, empfiehlt sich kaum.

42. Otto I (1903), 202; II, 326.

43. In der orphischen Kultgenossenschaft der Jobakchen in Athen wird der Priester

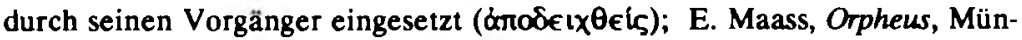
chen 1895, S.18. Aus Oxyrhynchos hört man von einem vorbezeichneten Schul-

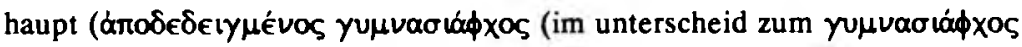
selber) P. Ox. 1242). Vgl. das von der römischen Gemeinde Erzählte: Eleutheros war bereits unter zwei Vorgängern Diakon (Euseb, $H E$ IV,22,2 f).

44. H. Volkmann, in Pauly-Wissowa 23, $1689 \mathrm{f}$.

45. Inscriptiones Graecae XI, 41299.

46. Graecus ignobilis (Livius 39, 8, 3).

47. Extra quam etc, dürfte sich nur auf Italia beziehen (Livius $39,18,7$ ).

48. $39,8,1-19,7$.

49. Sueton, Div. Julius 42.

50. Sueton, Augustus 32.

51. Plurimae factiones sub titulo collegi novi (Suet. Aug. 32). Vgl. Dig. 48, 19,30: novae sectae vel incognitae religiones.

52. Plinius, ep. $33.34 ; 92.93$.

53. Dagegen ist die von G. Krüger, Die Rechtsstellung der vorkonstantinischen Kirchen, Stuttgart 1935, S. 13 f. vertretene Ansicht, dass eine lex de collegiis in republikanischer Zeit existierte, eine reine petitio principii.

54. Vgl. Th. Mommsen, Römisches Strafrecht, Leipzig 1899, S. 578 f.

55. Livius $39,18,8 \mathrm{f}$.

56. Geschichte der juristischen Person I, München 1933.

57. S. Anm. 53

58. So Mommsen, Römische Geschichte V, 523; Ges. Schriften III, Berlin 1907, S. 410.

59. Vgl. V. Schnorr, S. 244 f.; insbes. $254 \mathrm{ff}$., Krüger, S. $69 \mathrm{ff}$. - Das Auflösungsdekret des Jahres 59 n. Chr. (Tacitus, Ann. 14, 17 : collegia quae contra leges instituerant) zeigt, dass solche Kollegien existierten und die Behörden sich veranlasst sahen, dem Wildwuchs zu steuern, ohne dass doch eine bestimmte gesetzliche Grundlage (darum Mehrzahl leges) vorhanden gewesen wäre. Die verschiedenen Massnahmen sind als Versuche anzusehen, Ordnung in gewohnheitsrechtliche Verhältnisse zu bringen. Es sind zwei Arten von Genossenschaften zu unterscheiden, solche, die von vorne herein ihre Einrichtung obrigkeitlichem Eingreifen verdanken (v. Schnorr, S. 380) und solche, die sich frei gebildet 
haben (vgl. W. Liebenam, Zur Geschichte und Organisation des römischen Vereinswesens, Leipzig 1890, S. 236). Was die letzteren anbelangt, so ist ein Unterschied zu machen zwischen den allgemeinen Erwägungen der Behörden und dem Zustand, sobald eine Anzeige erstattet war und das quaerere begann. Es sind ebenso zwei Arten von collegia illicita zu unterscheiden, eine, die bloss nicht autorisiert und darum nicht im eigentlichen Sinne illicit ist (Liebenam, $S$. 236; Krüger, S. 60) und einer anderen, die sich durch bestimmte Handlungen gegen die Gesetze vergangen hatte.

60. Livius $39,8,4$.

61. 60, 4-61, 3. Es folgen nur noch die Schlussermahnungen und die Grüsse. Zur Erklärung der Stelle vgl. Chr. Eggenberger, Die Quellen der politischen Ethik des 1. Klemensbriefs, Zürich 1951.

62. 1.3 und an vielen anderen Orten.

63. W. Mangold, De ecclesia primaeva pro Caesaribus ac Magistratibus Romanis preces fundente, Bonn 1881.

64. So wohl F. Overbeck, der seine Zweifel hinsichtlich der 'Ursprünglichkeit des ganzen Gebets' ausdrückt (Theol Lit Zeitung (1877) 286) und damit gegen A. Harnack Stellung nimmt, der in dem Kapitel eine' 'treue Reproduktion des römischen Kirchengebets' sah, um allerdings hinzuzufügen: im Gebet für die Obrigkeit 'darf man vielleicht die Hand des Briefstellers erkennen, der bekanntlich breite Ausführungen liebt' (Theol. Lit. Zeitung 1 (1876) 103). Zur Erörterung der Frage vgl. W. Wrede, Untersuchungen zum Ersten Klemensbriefe, Göttingen 1891, S. 57.

65. 7,$6 ; 0$, iff; $10,2,7 ; 19,1 ; 63,1$.

66. 1. Kl. 6.i f deutet nur an und ist allgemein formuliert. Das braucht jedoch nicht die Erklärung notwendig zu machen, die K. Heussi (Die römische Petrustradition in kritischer Sicht, Tübingen 1955, S. 20 f) vorschlägt.

67. 5,5 (vgl die von O Cullmann, Petrus, Stuttgart 1952, S. 100 ff. vorgenommene Erklärung der Stelle. Eifer und Neid hat es seit Kain und Abel gegeben (4.2). Grosse Städte uns mächtiger Völker sind dadurch zu Fall gekommen (6.4). Der Verfasser spannt diesen grossen Bogen, damit niemand auf den Gedanken kommt, die Unruhe in Korinth einseitig den Christen zu last zu legen.

68. Ob der Brief mehr einen privaten oder amtlichen Charakter habe, darüber sind Erörterungen zwischen R. Knopf (Handbuch, Ergänzungsband insbes. S. 119), A. v. Harnack (Einführung in die alte Kirchengeschichte Leipzig 1929, S. 88 ff.), B. Altaner (Kleine patristische Schriften Berlin 1967, S. 537 f.) und F. R. van Cauwelaert (L'intervention de l'église de Rome à Corinth vers 1'an 96, RHEccl. $31,1935,267 \mathrm{ff}$.) gepflogen worden. 
69. Etwas anders Wrede, S. 21.

70. Die tatsächliche Leitung der Gemeinde hat nicht in einer Hand gelegen. Noch Marcion steht in Rom den Presbytern gegenüber (Hippolyt, Syntagma; vgl. A. v. Harnack, Marcion, Leipzig 1924, S. 4) - von einem Bischof ist nicht die Rede. Zur späten Ausbildung des monarchischen Episkopats in Rom s. zuletzt P. Lampe, Die stadtrömischen Christen 1987, S. 334 ff.).

71. Hist. Eccl. V, 6.

72. So noch E. Caspar in seiner glänzenden Untersuchung Die älteste römische Beschofsliste, Berlin 1926 [ = Schriften der Köningsberger Gelehrten Gesellschaft, 2].

73. So Caspar, S. 175.

74. Bei Rufin ist Eleutheros ausgelassen. Soter wird gleichwohl als der zwölfte bezeichnet. Er ist es auch, wenn man die Apostel selbst mit einrechnet. So wird die Liste zu einer solchen apostolischer Sukzession im eigentlichen Sinne umgestaltet.

75. Ob die Sukzessionslisten in Const. Ap. 7, 46 in diesen Zusammenhang gehören? Für Jerusalem, Caesarea und Smyrna werden drei Namen genannt, für die anderen Orte einer oder zwei. Und wie steht es um die kurzen Sukzessionslisten der Gnostiker?

76. Davon zu unterscheiden sind die Gesichtspunkte bei der geistigen Auseinandersetzung. Daher rührt es, dass in den Schriften der Apologeten zwar der Altersbeweis und die Geordnetheit der christlichen Gemeinden eine Rolle spielt, nicht aber die Sukzession der Häupter.

77. Vgl. Minucius Felix, Octavius 6,3.: die vetustas verleiht Ehrwürdigkeit.

78. So manche Stelle im N.T. mag von daher Licht erhalten; etwa Act 17. Paulus wird nach seiner Predigt zwangsmässig auf die Gerichtsstätte des Areopags

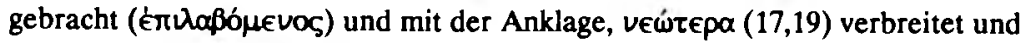
Fremdes eingeführt zu haben, konfrontiert. Er begegnet ihr mit der Behauptung der Bekanntheit von Elementen seiner Botschaft in dieser Stadt, und überhaupt in der griechischen Welt. Der Verfasser der Apostelgeschichte gibt an anderem Orte Kredentien für Paulus selber: 22, 3 und für seine Verkündigung des rarpúos Beós $(24,14)$.

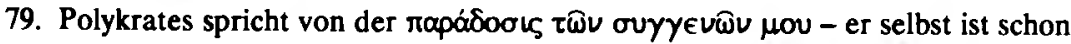
der achte (H.C. V, 24, 6) und hebt damit den Schleier über Verhältnissen, die denen in der erwähnten Gemeinde auf Delos vielleicht nicht unähnlich waren (die Generationenfolge seit der Gründung der Gemeinde ist sicher kürzer gewesen, als die Zahl acht anzeigt. Aber es lässt sich denken, dass jüngere Brü- 
der des Gemeindeleiters zu Häuptern von Tochtergemeinden bestimmt wurden).

80. Darüber beachtenswerte Erwägungen $\mathrm{H}$. Frhr. von Campenhausens, Lehrerreihen und Bischofsreihen im 2.Jahrhundert, in In Memoriam Ernst Lohmeyer, Stuttgart 1951, S. $240 \mathrm{ff}$. 